\title{
La comunicación política en Internet: el caso de \#RedAMLO en México
}

\author{
Political communication on Internet: \\ the case of \#RedAMLO in Mexico
}

\author{
Raúl Anthony Olmedo-Neri \\ Universidad Tecnológica de México - UNITEC MÉXICO- Campus En Línea \\ raulanthonyn@yahoo.com.mx \\ https://orcid.org/0000-0001-5318-0170
}

\begin{abstract}
Resumen
El presente trabajo analiza las modificaciones que las redes sociodigitales han generado dentro del campo de la comunicación política, particularmente la construcción de redes de interacción-comunicación mediante la creación de hashtags como el caso de \#RedAMLO en Twitter. Así, este trabajo tiene como objetivo identificar la red de interacción-comunicación que se han formado mediante el hashtag \#RedAMLO, para analizar su estructura, flujo de información y aquellos nodos que se posicionan estratégicamente en dicha red.

Para ello, se ha empleado el Análisis de Redes Sociales (ARS) como cuerpo metodológico y se ha recurrido a la minería de datos para recopilar, sistematizar, depurar y analizar la metacomunicación de dicha red.

Entre los principales resultados se encontró y visualizó una red conformada por 8586 nodos, mismos que son en buena medida ciudadanos que potencian su visibilidad y simpatía política a través de esta plataforma sociodigital. Esto es parte de un proceso más amplio donde una mayor visibilidad implica una descentralización de la opinión pública y una táctica política para mostrar la base que sustenta a la actual administración.

No obstante, dentro de los cinco nodos más relevantes por la cantidad de vínculos, dos presentan una lógica de funcionamiento similar a la de cuentas falsas o bots, por lo que el trabajo también identifica que en estas redes de orden político-ciudadano también intervienen elementos propios del espacio virtual derivado de sus lógicas operativas e intereses particulares.
\end{abstract}

\section{Palabras clave}

Hashtag, Twitter, comunicación, redes, política, imagen.

Forma sugerida de citar: Olmedo-Neri, R. (2021). La comunicación política en Internet: el caso de \#RedAMLO en México. Universitas, 34, pp. 109-130. 


\begin{abstract}
This paper analyzes the modifications that socio-digital networks have generated within the field of political communication, particularly the construction of interaction-communication networks through the creation of hashtags, such as \#RedAMLO on Twitter. Thus, this work aims to identify the interactioncommunication network that has been formed by the hashtag \#RedAMLO, to analyze its structure, information flow and those nodes that are strategically positioned in said network.

For this, the Social Network Analysis (SNA) has been used as a methodological body and data mining has been used to collect, systematize, debug, and analyze the metacommunication of said network.

Among the main results, a network made up of 8586 nodes was found and visualized, which are largely citizens who enhance their visibility and political sympathy through this socio-digital platform. This is part of a broader process where greater visibility implies a decentralization of public opinion and a political tactic to show the base that sustains the current administration.

However, within the five most relevant nodes due to the number of links, two have an operating logic similar to that of fake accounts or bots, which is why the work also identifies that elements are also involved in these citizen-political networks. characteristic of the virtual space derived from its operational logics and particular interests.
\end{abstract}

Keywords

Hashtag, Twitter, communication, networks, politics, image.

\title{
Introducción y marco teórico
}

Una característica emergente, que se consolida con rapidez en la sociedad contemporánea, es la vivencia/construcción del mundo en y a través de las pantallas y las plataformas que en ellas se consolidan. Facebook, Twitter, Instagram y las aplicaciones de interacción social se han convertido en elementos indisociables de la vida cotidiana del siglo XXI. Ellas no solo están en la red, sino que son en esencia interfaces pensadas en esa arquitectura reticular, ya que su finalidad está orientada a la conectividad como cultura (Van Dijck, 2016). Su uso, que en un principio estuvo marcado por el entretenimiento, se ha ampliado a procesos laborales, prácticas culturales, dinámicas económicas, procesos de interacción social y estrategias políticos, por lo que su estudio no debe limitarse a una cuestión particular, sino en el 
conjunto de modificaciones y reconfiguraciones que emanan de su irrupción en la vida cotidiana.

No obstante, este proceso de incorporación y apropiación social de la tecnología no es nuevo, ni único. De hecho, esto ha sucedido con cada nuevo medio de comunicación que se desarrolla e inserta en la vida social; así ocurrió con el periódico, la radio, la televisión y la industria cinematográfica (García-Calderón \& Olmedo-Neri, 2019). Sin embargo, lo realmente novedoso de las plataformas sociodigitales es el corto tiempo en que fueron incorporadas por parte de los individuos en su cotidianidad, es decir que el proceso de apropiación (Crovi, 2012) fue más rápido que el de otros medios de comunicación.

La llegada de Internet y las plataformas sociodigitales no implica la desaparición de sus antecesores per se, sino más bien un proceso de asimilación, adaptación y descentralización en el sistema mediático y la opinión pública de dicha sociedad; no hay exclusión a priori, sino un proceso de conectividad, de vinculación y de convergencia mediática que da paso a un nuevo escenario tecnológico y comunicativo para la participación ciudadana en el ámbito político. De allí que Lipovetsky y Serroy (2009, p. 271) hablen de una malla de pantallas que "ha transformado nuestra forma de vivir, nuestra relación con la información, con el espacio-tiempo, con los viajes y el consumo" y donde la propia interacción social se ve mediada, mediatizada y condicionada por las novedosas formas de socialización que allí se desarrollan (Boyd, 2011; Quinn \& Papacharissi, 2018).

De todas esas posibles combinaciones, la vinculación entre comunicación y política adquiere relevancia dadas las nuevas ventajas y desventajas que se manifiestan con el uso de Internet y las plataformas sociodigitales para fines políticos.

Si bien la relación mediada entre comunicación y política ha estado presente desde sus inicios, es con la Modernidad en que "ambas han ayudado a la organización del Estado y al desarrollo de sus potencialidades políticas, económicas y sociales" (Reyes-Montes et al., 2011, p. 86). Así es cómo surge el campo interdisciplinario de la comunicación política, el cual "estudia las dimensiones contradictorias y complementarias que se presentan en la política, información y comunicación en sistemas democráticos" (ReyesMontes et al., 2011, p. 90)

En términos generales, la comunicación política se constituyó a inicios de los años cuarenta y cincuenta del siglo XX, específicamente con el tér- 
mino de la Segunda Guerra Mundial (Reyes- Montes, 2007; Mendieta-Ramírez \& Estrada-Rodríguez, 2017). Desde 1970, esta área de conocimiento parte de "concebir a los medios de comunicación como un factor trascendental y poderoso en la forma de pensar y actuar de los individuos" (ReyesMontes, 2007, p. 109).

Lo anterior es relevante dado que la comunicación política es el resultado histórico de la interconexión entre la esfera política y la mediática, donde ambas partes han interactuado en una relación simbiótica. Este proceso se consolidó cuando los medios de comunicación masiva se posicionaron como un elemento más para evaluar el nivel de democracia en un Estado-Nación. A partir de ello se construyó la idea de "recurrir cada vez más al soporte de los mass-media como canales privilegiados del contacto con los electores y de la reactivación de la participación política" (Grossi, 1985, pp. 152-153).

Sin embargo, la llegada de las redes sociodigitales cambió ese estado de cosas ya que generó dos modificaciones relevantes: la primera refiere a la descentralización de los medios de comunicación tradicionales como productores de la opinión pública, y la consecuente redistribución de la participación ciudadana en el espacio virtual (Mathias, 2012). Mientras que la segunda modificación es más profunda ya que las novedosas formas de articular estrategias políticas fuera de ciclos electorales o basados en los procesos administrativos del sistema democrático, amplía la participación ciudadana de manera permanente, convirtiendo a Internet y las plataformas como herramientas "muy útiles para ayudar a fomentar un poder cívico subjetivo y un mayor sentido de participación basado en la comunicación horizontal" (Dahlgren, 2012, p. 52).

Entonces la participación ciudadana en el ámbito político ya no se reduce a un voto en una fecha establecida, sino en una constante intervención en la opinión pública a favor o en contra de una postura ideológica o política con la que comulga o diverge. Esta ampliación en la forma de participar políticamente tiene un carácter subjetivo y de interacción al compartir, denunciar, comentar, reaccionar o participar en el contenido que fluye permanentemente en las plataformas como Facebook y Twitter.

Por ello, en las redes sociodigitales se "da lugar a la acción creativa y autónoma de los usuarios, de modo que aquellas fuerzas pueden verse consolidadas, negociadas o desafiadas" (Jorge, 2014, p. 272). Ante este nuevo contexto, los conglomerados mediáticos se ven obligados a realizar una convergencia digital, es decir, en la creación de perfiles/cuentas en cada una 
de esas plataformas para mantener e incrementar su condición 'masiva'. Su fuerza y legitimidad es desafiada porque la estructura reticular, oblicua y multidireccional que existe en la red hace factible que se presenten visiones, opiniones, ideas e información que pueden entrar en conflicto con aquella producida en esos medios masivos de comunicación, dando lugar a un nuevo escenario donde "los ciudadanos, inmersos en las redes digitales, tienen más recursos que nunca para deliberar” (Trejo-Delarbre, 2015, p. 22).

A partir de esa horizontalidad de participación y descentralización de la información masiva, la posición estratégica de periodistas y líderes de opinión se reduce a la función usuario/usuario en las plataformas (Olmedo-Neri, 2020), donde "la red tiende a facilitar nuevas formas de comunicación, tanto horizontal, como vertical, que fomentan las discusiones en el espacio público" (Gómez- Castellanos, 2011, p. 75). Así pues, este lugar sin materialidad espacial, pero con una delimitación orgánica, se configura como el nuevo escenario donde las relaciones entre comunicación, participación y poder se modifican sustancialmente dada la infinita pluralidad de fuentes de información y la creciente intervención de los ciudadanos en cuestiones de muy diversa índole, entre ellas la política a nivel multiescalar.

El ejemplo más relevante del aprovechamiento de este nuevo escenario para la acción/participación/interacción política es la campaña electoral de Barack Obama en Estados Unidos, la cual "fue percibida como la primera en combinar el uso de las redes sociodigitales con la movilización sobre el terreno de una manera más amplia y efectiva" (Baldwin-Philippi, 2018, p. 528). El impacto mostrado en esa campaña dio paso a un prolífico uso de estas redes por parte de la clase política para materializar la victoria, ya que Obama pudo vincular positivamente a los ciudadanos, quienes formaron parte activa en la recolección de fondos, con la difusión de los mensajes hacia grandes sectores poblacionales, especialmente la juventud estadounidense.

Este 'giro' en la forma de hacer política no solo impactó en las campañas, sino en la propia forma de construir y mantener una imagen adecuada en la esfera pública que pueda mantenerlo en el poder. Entonces este giro es de corte simbólico y comunicativo en tanto se asimila que "no se trata ya de convertir ideológicamente a los ciudadanos, se trata de vender — un producto - con la mejor envoltura posible" (Lipovetsky, 1990, p. 225). Lo anterior obedece a un proceso paulatino que desplaza el factor racional de las propuestas que ofrecen, para pasar a un factor emotivo que genere un vínculo más fuerte y duradero que el periodo en que se posee el cargo público. 
Todo lo anterior genera, por tanto, un nuevo escenario que modifica de una u otra manera la forma de hacer política, lo cual se ve reflejado no solo en las campañas de los que aspiran a un cargo dentro del Estado, sino en la (re)configuración simbólica de toda aquella persona que desea ostentar un puesto público. Además de ello "la actual metamorfosis de los medios de comunicación está teniendo un profundo impacto en las condiciones de la participación y en la dinámica de la democracia" (Dahlgren, 2012, p. 51).

Lo anterior no significa que con internet exista una mayor pluralidad, ni mucho menos que el debate sea consensado. Por el contrario, la también llamada red de redes se convierte en un lugar donde la polarización se encuentra presente porque suponer una mayor conectividad e interacción no significa que la gente cambie su postura política de manera radical. En términos de redes, se replica la homofilia (Lozares \& Verd, 2011), es decir la tendencia de las personas a agruparse con otras personas de acuerdo con sus intereses al grado de construir burbujas donde fluye el mismo sentido político y que en ocasiones se ve en conflicto al colisionar con otras esferas cohesionadas a partir de elementos ideológicos o políticos contrarios.

Además de ello, esta amplitud también deriva en la incursión de nuevos elementos que amplían sus efectos disruptivos: las noticias falsas y los bots se configuran como recursos que también se integran de manera general en internet, pero con particular profundidad en el ámbito de la comunicación política ya que pueden jugar a favor o en contra de un partido, un político o una administración.

Por ello, ante las ventajas y desventajas mencionadas aquí, resulta menester analizar un caso particular para identificar cuál es la red que se configura y de qué manera las formas de participación y los retos del contexto digital se encuentran presentes, exponiendo la complejidad social. El caso de la \#RedAMLO resulta útil para analizar cómo las estrategias políticas tienen un carácter ciudadano, pero también comunicativo en el ámbito político.

Además de \#RedAMLO se han construido otros hashtags como \#AMLOVE \#Chayoteros, \#PrensaFifí y \#AMLOvers durante la administración de Andrés Manuel López Obrador (AMLO) como parte de estrategias políticas para 'mostrar' el apoyo de los usuarios simpatizantes en Twitter. Desde tiempo atrás se viene advirtiendo que en este tipo de estrategias es plausible el uso de bots o cuentas falsas, e incluso se ha llegado a identificar en algunos casos cómo es que esas cuentas orquestan o incrementan la visibilidad del actual Presidente de México (Chávez, 2020; Tajonar, 2020), por lo que el análisis de esta red permite que se evidencien tanto las estrategias 
ciudadanas como aquellas cuentas que por su lógica de interacción en Twitter pueden ser consideradas como cuentas falsas.

Para ello el presente trabajo propone el Análisis de Redes Sociales (ARS) como método que puede ayudar a analizar esta estructura reticular de interacción-comunicación (Olmedo-Neri, 2019) que se desarrolla alrededor de dicho hashtag. La red de interacción-comunicación está conformada por enlaces que se generan entre dos o más nodos mediante la interacción que desarrollan con un producto creativo específico (meme, imagen, hashtag, video, link, entre otras), con el cual pueden reforzarse relaciones de amistad, crear nuevos vínculos o destruir los ya existentes. Estas redes de interacción-comunicación no están basadas necesariamente en la red de vínculos que posea un individuo per se, sino que pueden motivar nuevos vínculos con mayor o menor perdurabilidad mediante la información con la que interactúan en un momento dado.

Así, estas redes que se estructuran conforme al flujo de información son particularmente especiales en el espacio digital ya que pueden reforzar los lazos de amistad o las lógicas de operación (seguir/ser seguido) a partir de ese contenido, generar nuevos vínculos con otros usuarios o modificar los que ya se tenían previamente.

De esta manera, la noción de red adquiere relevancia en su sentido comunicativo ya que ella se estructura por la información y el significado que acompaña a \#RedAMLO en Twitter. Por tanto, es posible que se organicen y visualicen aquellas redes políticas de defensa/promoción/ataque hacia o desde un político o partido por su actuar en la constante esfera política del país.

\section{Metodología}

El Análisis de Redes Sociales (ARS) es un método que:

Pretende analizar las formas en que individuos u organizaciones se conectan o están vinculados, con el objetivo de determinar la estructura general de la red, sus grupos y la posición de los individuos u organizaciones singulares en la misma. (Sanz, 2003, p. 23)

La factibilidad de emplear este método para el análisis del hashtag \#RedAMLO deriva de sus aportes al estudio estructural de la red y el flujo de información que allí se desarrolla. Desde la estructura, el ARS permite identificar y analizar la red en sí, es decir, el número de participantes (nodos) que 
intervienen y los vínculos que se generan entre dichos nodos; mientras que a nivel informativo aporta elementos para rastrear y observar el contenido que permite que dos usuarios, que pueden o no tener una vinculación per se, se encuentren dentro de la infinitud de la plataforma Twitter.

El ARS surge de aportaciones de la sociología, la antropología y las matemáticas, entre otras, con la finalidad de construir indicadores que permitan medir la cohesión, la posición estratégica de los nodos (usuarios/ individuos) y la cantidad de vínculos (relaciones entre dos o más nodos) que estructuran la red. Su uso en el ámbito digital adquiere relevancia desde los inicios propios del internet (Rogers, 2018) y con el paso del tiempo se han diversificado sus objetos de estudio: desde las redes egocéntricas que posee un individuo dentro de una plataforma, pasando por el impacto de una campaña electoral en el ámbito digital mediante el uso de la nube (Peirone, 2012), hasta el análisis de los procesos de convergencia mediática que realizan movimientos sociales (Olmedo-Neri, 2019) para incrementar su visibilidad en el espacio público digital.

En este trabajo se ha utilizado el software NodeXL para la recolección de la información y el programa Gephi para la visualización de la red y el cálculo de los indicadores. Los procesos de recolección están vinculados a los alcances de la investigación y a la temporalidad de los hechos que promueven el uso o no de un recurso como el hashtag, un meme o un video, por lo tanto, su recolección y análisis deben estar contextualizados temporal y espacialmente con el momento en que se utiliza ya que tiene un significado crucial.

En este sentido, la red que se analiza está estructurada alrededor del hashtag \#RedAMLO en Twitter ${ }^{1}$, este recurso posee un carácter de trazabilidad, es decir, puede ser rastreado mediante NodeXL para identificar aquellos nodos (usuarios/perfiles) que han publicado, compartido o comentado algo sobre dicho hashtag.

En términos de delimitación temporal el presente estudio ha recopilado las relaciones de interacción-comunicación desarrolladas alrededor del hashtag \#RedAMLO del 6 al 30 de abril de 2020, dado que en este periodo se incrementó el uso de hashtags a favor del Presidente de México y con ello los cues-

1 En los inicios de la aplicación del ARS en el espacio digital era posible hacer este tipo de análisis en Facebook, sin embargo, con la modificación de sus políticas y términos de uso, esta posibilidad se eliminó, dando paso a que este tipo de análisis se usara en otras plataformas como Twitter, YouTube y Flickr, por ejemplo. 
tionamientos a estos recursos para 'inflar' la popularidad de AMLO (Chávez, 2020; Tajonar, 2020). De allí que \#RedAMLO se configura como una estrategia política para mostrar la simpatía y apoyo al mandatario en momentos particulares y donde también está el latente uso o participación de cuentas falsas o bots.

En cuanto a la delimitación espacial, se han analizados aquellos usuarios que poseen una ubicación dentro del territorio mexicano, con la finalidad de centrar el análisis al territorio nacional, dado el cargo político que posee Andrés Manuel López Obrador (AMLO).

Una vez que se ha identificado el elemento sujeto a trazabilidad y las delimitaciones temporales y espaciales, es necesario establecer los indicadores que serán utilizados para analizar tanto la estructura como la lógica operativa de la red que subyace en el hashtag \#RedAMLO. En la siguiente tabla se muestran los indicadores a emplear.

\section{Tabla 1 \\ Indicadores de red}

\begin{tabular}{|l|l|}
\hline \multicolumn{1}{|c|}{ Indicador } & \multicolumn{1}{c|}{ Descripción } \\
\hline Tipo de red & $\begin{array}{l}\text { Puede ser dirigida (que se conoce la dirección del vínculo entre dos nodos) } \\
\text { o no dirigida (que solo se limita a identificar la presentación del vínculo). }\end{array}$ \\
\hline Densidad de red & $\begin{array}{l}\text { "La densidad de un grafo es la relación entre el número de líneas existentes } \\
\text { divido por el número de líneas posibles" (Paniagua, 2012,p. 37). }\end{array}$ \\
\hline Nivel de grado & $\begin{array}{l}\text { Es la cantidad de vínculos que posee un nodo dentro de la red. Si la red es } \\
\text { de tipo dirigida, el nivel de grado puede desglosarse en grado de entrada } \\
\text { (aquellas interacciones de uno o más nodos respecto a una publicación } \\
\text { específica de un usuario) y grado de salida (aquellas publicaciones que } \\
\text { emanan de un nodo). }\end{array}$ \\
\hline Grado de intermediación & $\begin{array}{l}\text { Con este indicador se alude a un atributo de dispersión y concentración } \\
\text { donde fluye la información y desde donde pueden incrementar su } \\
\text { visibilidad si así se le desea (Hanneman, 2000). }\end{array}$ \\
\hline
\end{tabular}

Fuente: Elaboración propia.

Finalmente, con estos indicadores es posible identificar la estructura y la lógica interna que posee la red, así como aquellos nodos que se posicionan estratégicamente en dicha estructura reticular, sea por la cantidad de vínculos que posean o por su capacidad de difusión de información en dicha estructura reticular. 


\section{Resultados}

El hashtag \#RedAMLO se convierte en un ejemplo del aprovechamiento de las lógicas operativas de Twitter para convocar y articular en Twitter la simpatía de ciudadanos a favor de la administración federal que encabeza Andrés Manuel López Obrador (AMLO) desde su triunfo electoral en México en junio de 2018.

La red es de tipo dirigida, lo que significa que se conoce el origen y el destino de cada vínculo. Esto es particularmente importante ya que al conocer la direccionalidad es posible explicar la lógica funcional del flujo de información de la red, es decir, quién tiene mayor capacidad para difundir información y quién posee una posición estratégica, por ejemplo.

A nivel estructural, la red está constituida por 8586 nodos y 26649 vínculos. De la depuración de datos realizada se encontró que los lugares donde hubo más publicaciones fue la Ciudad de México, Monterrey y Guadalajara. La siguiente figura muestra la red a partir del grado de entrada.

\section{Figura 1}

\section{\#RedAMLO}

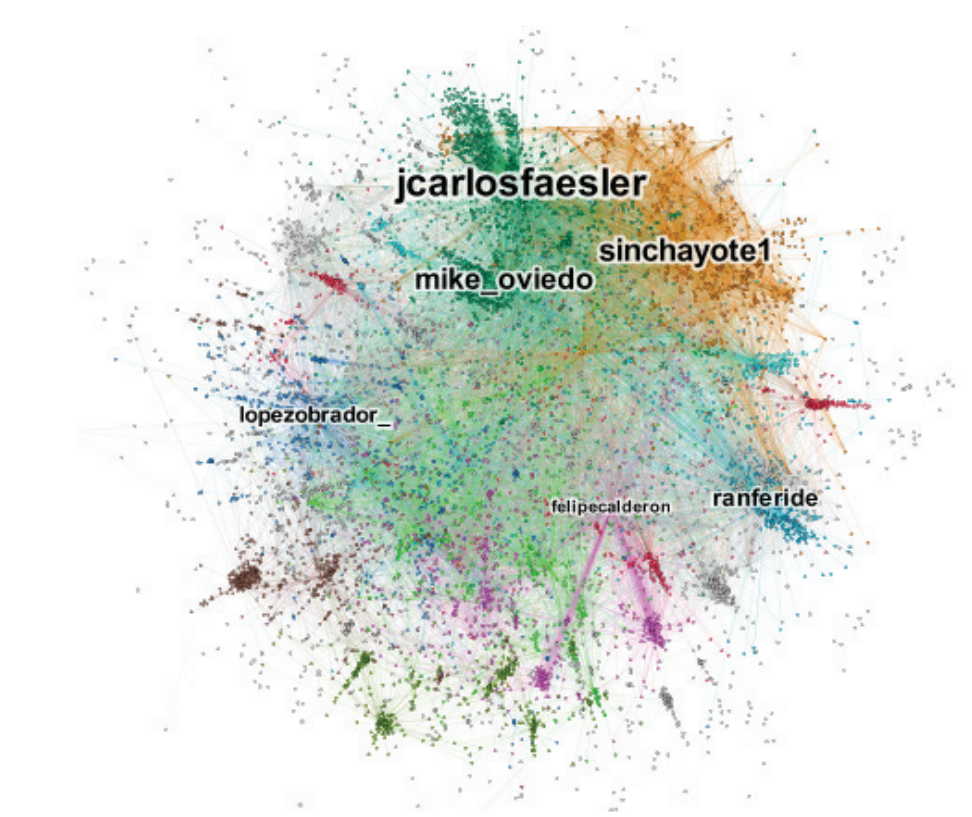

Fuente: Elaboración propia con datos de Gephi. 
La densidad de esta red es $0.001 \%$, lo cual explica que esta red posee una débil cohesión entre sus nodos, sin embargo, ello responde a la finalidad del hashtag: llegar a la mayor cantidad de personas posible, independientemente de que dicha vinculación pueda perdurar después de emplear ese recurso en un momento particular.

La red está presentada bajo el algoritmo ForceAtlas2 de Gephi; los nombres presentes en dicha red corresponden a los usuarios con mayor nivel de grado, es decir, aquellos nodos que poseen la mayor cantidad de vínculos en la red. Se ha destacado el caso de la cuenta oficial del expresidente Felipe Calderón (2006-2012), con la finalidad de mostrar que esta red no solo tiene un fin reivindicar la imagen de AMLO en la esfera pública, sino que también denuncia aquellos gobiernos cuyas acciones contribuyeron de cierta manera al contexto actual en el país; además de ello, la relación tensa entre AMLO y Felipe Calderón existe desde las elecciones presidenciales del 2006, donde Calderón salió elegido con un margen muy pequeño y criticado por una parte de la sociedad mexicana.

En la siguiente tabla se observan los indicadores de los usuarios con mayor cantidad de vínculos.

\section{Tabla 2}

Nodos con mayor nivel de grado

\begin{tabular}{|l|l|l|l|l|l|}
\hline \multicolumn{1}{|c|}{ Nodo } & \multicolumn{1}{|c|}{ Rol } & $\begin{array}{c}\text { Grado } \\
\text { Entrada }\end{array}$ & $\begin{array}{c}\text { Grado } \\
\text { Salida }\end{array}$ & $\begin{array}{c}\text { Nivel de } \\
\text { Grado }\end{array}$ & Intermediación \\
\hline jcarlosfaesler & Simpatizante de AMLO & 1525 & 3 & 1528 & 1078095 \\
\hline Sinchayote1 & $\begin{array}{l}\text { Página de contenido a } \\
\text { favor de AMLO }\end{array}$ & 903 & 223 & 1126 & 1699036.4 \\
\hline Mike_oviedo & Simpatizante de AMLO & 1001 & 4 & 1005 & 663005.34 \\
\hline Ranferide & Simpatizante de AMLO & 663 & 1 & 664 & 0 \\
\hline Lopezobrador_ & Presidente de México & 574 & 0 & 574 & 0 \\
\hline
\end{tabular}

Fuente: Elaboración propia con datos de Gephi.

Entender la operatividad de los indicadores permite reconocer la lógica funcional de dicha red. En este caso, al conocer la direccionalidad del vínculo, es decir, de dónde se genera y hacia qué nodo se dirige, es posible desagregar el nivel de grado en dos subgrupos; así, "mientras que el grado de 
entrada hace alusión a los vínculos referidos hacia un nodo, el grado de salida alude a los vínculos que los nodos crean" (Olmedo-Neri, 2019, p. 123).

De esta manera se puede observar que, @jcarlosfaesler publicó tres tuits en los que se incluyó el hashtag \#RedAMLO, mientras que este usuario tuvo 1525 interacciones con ese hashtag; dichas interacciones pueden ser derivadas de sus publicaciones o por ser etiquetado en tuits donde se encuentra dicho hashtag. El caso de @sinchayote1 es similar, dado que mientras este perfil publicó durante el mes de abril 223 tuits con este hashtag, dicha actividad generó 903 interacciones con otros usuarios. En estos dos casos se puede observar que los indicadores no están correlacionados de manera directa, por lo que un grado de salida alto no siempre corresponderá con un grado de entrada similar y viceversa.

De hecho, esta autonomía relativa de los indicadores encuentra su lógica no en la red, sino en el usuario mediante la fortaleza de sus vínculos para generar una potencial difusión de lo que se publica desde su cuenta; es el factor social, simbólico y subjetivo el que permite que los indicadores se construyan alrededor de los nodos y consecuentemente en la red.

Destaca el caso de la cuenta oficial de Andrés Manuel López Obrador en tanto dicho nodo no publicó ningún tuit con dicho hashtag, pero esta cuenta fue etiquetada en 574 tuits, lo cual muestra que esta red, si bien está estructurada en torno a AMLO, su cuenta no es el centro directriz de esta red, sino que es uno de los elementos que le dan sentido simbólico, de articulación y comunicación en el espacio digital.

El nivel de intermediación dentro de este tipo de análisis adquiere relevancia dado que con él se puede observar el carácter diseminador de un nodo en cuanto a información se refiere. La intermediación muestra la cantidad de caminos en las que el nodo participa para vincular otros dos usuarios en la red; por ende, al tener un alto grado de intermediación, el nodo se posiciona estratégicamente como un hub (Barabási, 2011) es decir un nodo que puede diseminar con mayor rapidez la información dentro de la red debido a la cantidad y direccionalidad de los vínculos que posee.

En este sentido, el grado de intermediación de @jcarlosfaesler expone que este nodo participa en poco más de un millón de caminos para conectar a dos nodos dentro de la red; tanto @raferide como @lopezobrador_no poseen grado de intermediación dado que su interacción con el hashtag o con los otros usuarios no fueron relevantes dentro de la estructura reticular. 
Por tanto, cada indicador puede estructurar la red de manera diferente y por consiguiente reposicionar estratégicamente a los nodos existentes.

De esta manera, en la siguiente figura se muestran los cinco nodos con mayor grado de intermediación.

\section{Figura 2}

\#RedAMLO con grado de intermediación

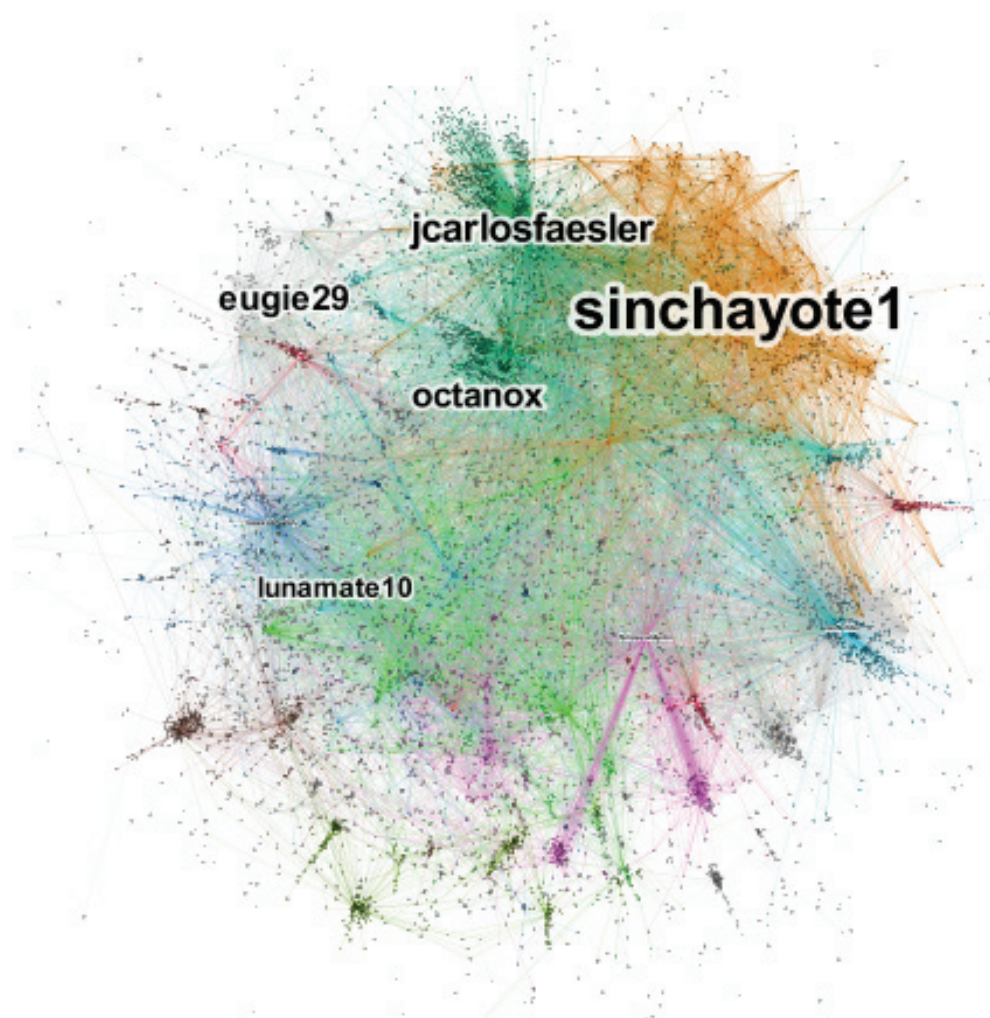

Fuente: Elaboración propia con datos de Gephi.

$\mathrm{Al}$ cambiar el indicador bajo el cual posicionar los nodos, estos pueden o no mantenerse como estratégicos. Como se observa en ese caso, algunos nodos se mantienen y otros más son desplazados; la siguiente tabla muestra los indicadores de dichos nodos. 
Tabla 3

Nodos con mayor grado de intermediación

\begin{tabular}{|l|l|r|r|r|r|}
\hline \multicolumn{1}{|c|}{ Nodo } & \multicolumn{1}{|c|}{ Rol } & $\begin{array}{c}\text { Grado } \\
\text { Entrada }\end{array}$ & \multicolumn{1}{c|}{$\begin{array}{c}\text { Grado } \\
\text { Salida }\end{array}$} & $\begin{array}{c}\text { Nivel de } \\
\text { grado }\end{array}$ & Intermediación \\
\hline Sinchayote1 & $\begin{array}{l}\text { Página de contenido a } \\
\text { favor de AMLO }\end{array}$ & 903 & 223 & 1126 & 1699036.4 \\
\hline Jcarlosfaesler & Simpatizante de AMLO & 1525 & 3 & 1528 & 1078095 \\
\hline Eugie29 & Simpatizante de AMLO & 9 & 64 & 73 & 1044914.9 \\
\hline Octanox & Simpatizante de AMLO & 73 & 7 & 80 & 1035494.4 \\
\hline Lunamate10 & Simpatizante de AMLO & 133 & 21 & 154 & 745660.9 \\
\hline
\end{tabular}

Fuente: Elaboración propia con datos de Gephi.

Bajo el presente panorama es posible observar la relativa autonomía de cada indicador, por lo que poseer una cantidad alta de publicaciones o interacciones no significa precisamente que se posea una capacidad de diseminación de la información en la red.

A la par del análisis estructural realizado, es necesario identificar las características de los contenidos que fluye en dicha red, con la finalidad de caracterizarla en términos simbólicos y comunicativos. Si bien, en las redes sociodigitales existe una libertad relativa para presentarse mediante una cuenta/perfil, también existe la posibilidad de trascender esa libertad para obtener diferentes, múltiples y a veces infinitos perfiles o cuentas de una misma persona; en otras ocasiones es posible identificar aquellas cuentas que son creadas de manera premeditada para sobredimensionar la presencia de un movimiento o acción política. Bajo esa lógica, se ha empleado el programa Botometer (2020) para identificar en términos analíticos la probabilidad que las cuentas que están estratégicamente posicionadas en la red puedan ser bots o cuentas falsas.

Dicho software tiene orígenes en la Universidad de Indiana y analiza "unas 1200 características para caracterizar el perfil de la cuenta, los amigos, la estructura de la red social, los patrones de actividad temporal, el lenguaje y el sentimiento" (Derecho a la Red, 2020); estos factores están creados a partir de modelos sobre cómo se comporta una cuenta falsa, por lo que mientras más alto sea el indicador, existe una mayor posibilidad de que dicha cuenta sea falsa o bot. 
En la siguiente figura se puede observar el resultado obtenido de cada uno de los nodos que se han posicionado estratégicamente en la \#RedAMLO tanto en el grado como en la intermediación.

Figura 3

Probabilidad de ser cuentas falsas o bots

\begin{tabular}{|c|c|}
\hline @Lunamate10 & A not found \\
\hline$>$ 9.릭 @octanox & $1 / 5$ \\
\hline$>$ DEUGIE29 & $3 / 5$ \\
\hline >f @lopezobrador_e & $1 / 5$ \\
\hline$>-0.2$ @RanferiDe & $1 / 5$ \\
\hline > Fild @Mike_oviedo & $2 / 5$ \\
\hline >chisite @SinChayote1 & $5 / 5$ \\
\hline >10 @JCarlosFaesler & $7 / 5$ \\
\hline
\end{tabular}

Fuente: Botometer (2020).

Cabe resaltar que esta herramienta solo muestra un resultado probabilístico a partir del análisis de las variables que han sido previamente establecidas, por lo que es necesario identificar otros elementos dentro de la cuenta como nombre de usuario, contenido en sus tuits o si hay un constante número de perfiles a etiquetar en cada publicación, por ejemplo, por lo que estar con plena seguridad de la falsedad de una cuenta implica un trabajo más profundo sobre cada uno de los perfiles, que sale fuera de los alcances propios de la presente investigación.

En términos de contenido, se puede observar que la \#RedAMLO tiene diversas funciones entre las que destaca la constante interacción entre los 
también autodenominados \#AMLOvers. En la siguiente figura se puede observar uno de los tuits que muestra la forma en que se establecen estos vínculos, mismos que configuran la red.

\section{Figura 4}

Tuit de@jcarlosfaesler

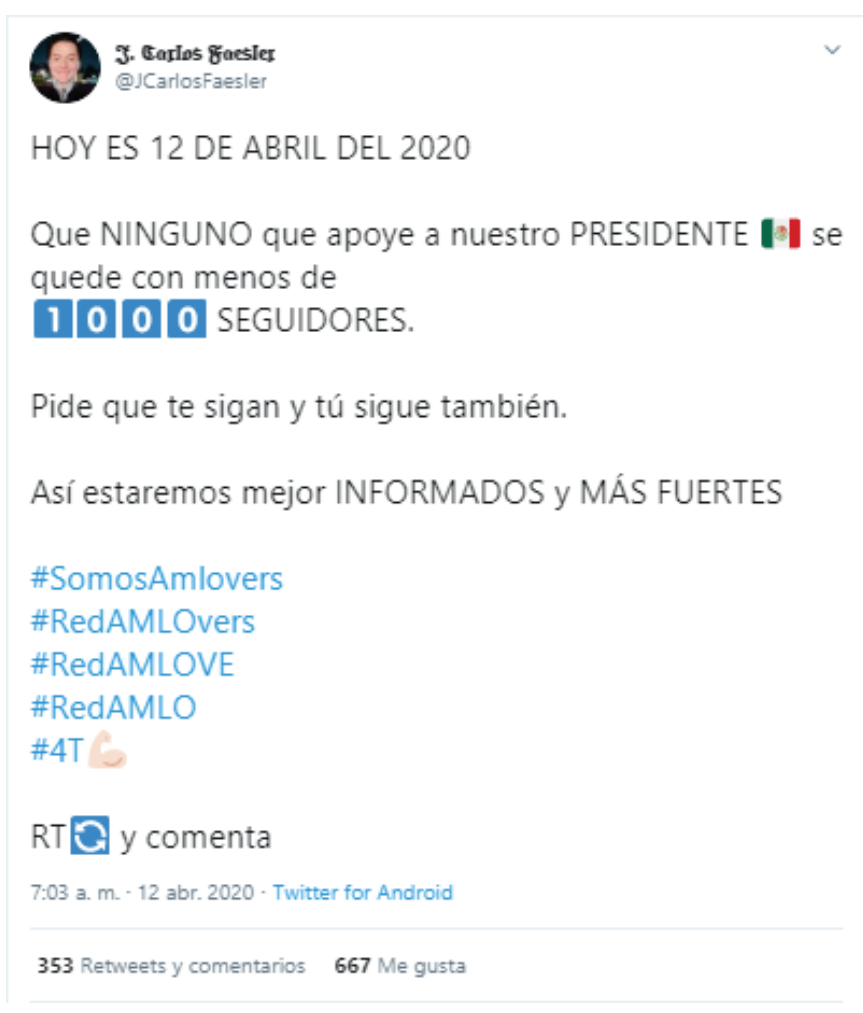

Fuente: @scarlosfaesler(2020).

De esta manera, las publicaciones dentro de esta red están enfocadas a mantener los vínculos entre sus integrantes, así como incrementar su visibilidad a partir de seguirse mutuamente y retuitear el contenido generado. Estas estrategias tienen la finalidad de incrementar la densidad de la red, mediante el establecimiento de vínculos operativos (seguir/ser seguido) en Twitter. Por otro lado, esta red se caracteriza por hacer de manera constante 
una vinculación comparativa entre AMLO y los presidentes anteriores, particularmente Felipe Calderón, con quien, como se ha mencionado, persiste una histórica relación tensa por cuestiones políticas y de poder. Este tipo de comparaciones tienen un impacto menor en cuestión de interacción, pero su presencia es contante, lo que muestra que esta red no solo se caracteriza por mantener sus vínculos, sino también por hacer una constante reafirmación positiva de la imagen y postura política de AMLO.

\section{Figura 5}

\section{Comparación sobre gestiones entre AMLO y Felipe Calderón}

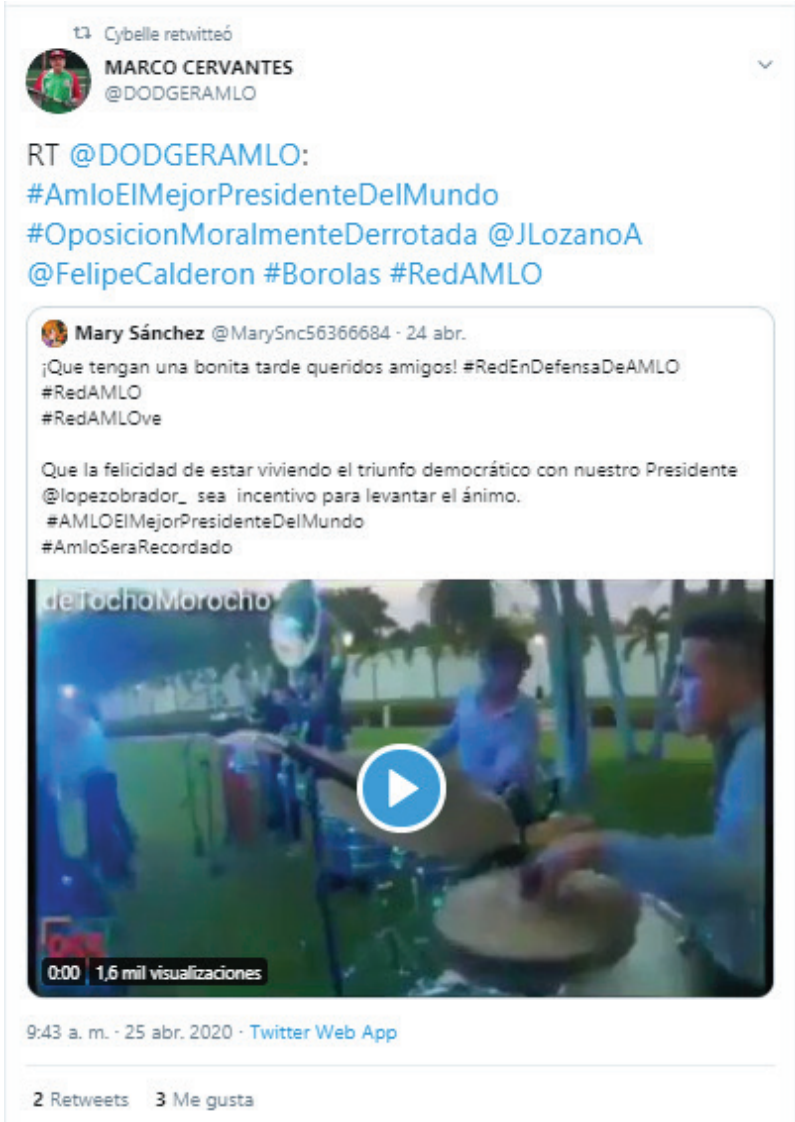

Fuente: @Dodgeramlo (2020). 
Finalmente, se puede observar que esa red cuenta con diversas finalidades, cada una establecida de acuerdo con los intereses que se generan en el contexto y la información que surge de aquellos usuarios que no concuerdan con la administración de AMLO.

\section{Conclusiones}

El presente trabajo ha analizado una red de usuarios que se estructura bajo el hashtag \#RedAMLO. Los resultados permiten mencionar que dentro de la red existen usuarios que se posicionan estratégicamente por el rol que juegan dentro y fuera de Twitter: mientras a nivel externo resulta relevante la posición del usuario en la opinión pública, a nivel interno influye la interacción que posea y los atributos que recibe de los vínculos que forman. De esta manera se puede decir que no basta con tener la firme convicción política de apoyo para ostentar un lugar estratégico y de incitación en la esfera digital, sino que va de la mano con estrategias y flujos de información dentro de la plataforma.

También resulta importante destacar la intervención de nuevos factores que no mediaban anteriormente: los bots o cuentas falsas que son generadas y administradas para simular una presencia o fuerza en el espacio digital se vuelven particularmente relevantes, dado que cuestionan a priori cualquier red generada en el espacio virtual. Independientemente de su origen, su finalidad puede ayudar a generar una percepción de la fuerza política que respalda a una administración, o también configurarse como un promotor de cuestionamiento y desprestigio al objetivo definido. Por ello, el abordaje de estos nuevos 'actores' en el ámbito de la comunicación política tiene un camino largo no solo a nivel teórico, sino también metodológico para su identificación dentro de grandes bases de información.

A nivel estructural la red se analizó bajo el ARS; con esto se observó que el rol de aquellos nodos con una posición estratégica dentro de dicha red son los simpatizantes de AMLO y algunas páginas que permanentemente están generando y difundiendo información a favor de Andrés Manuel López Obrador. A nivel operativo e informativo, estos nodos difunden contenido a favor de AMLO de manera constante, por lo que se puede observar cómo la participación de los individuos en el ámbito político ya no solo se restringe a los procesos electorales establecidos en la democracia mexicana, sino que se ha ampliado a una constante dinámica de reivindicación 
del político dentro del cargo público que ostenta; es una forma en que las redes de apoyo y simpatía política se digitalizan en el espacio virtual. Quizá, la idea de poder etiquetar al Presidente de México con la esperanza de ser leído por él, se convierte en la motivación más fuerte de generar estas redes de interacción-comunicación

Si bien estas formas de redes se encontraban presentes en el ámbito real concreto, con las plataformas sociodigitales dichas redes se amplían más allá del espacio en el que el individuo se desarrolla; la capacidad de encontrar a personas que concuerdan con una posición política aumenta con internet de la misma manera que el poder encontrar a alguien que no concuerda con dicha postura. Por ello, en el estudio abordado se observa cómo con estos nuevos desarrollos tecnológicos la actividad política se fuerza a exponer un mayor carácter comunicativo que va más allá de los estudios de opinión antes, durante y después de un proceso político-electoral, para dar paso a la digitalización de las redes sociales de simpatía política, alianza electoral y vinculación comunicativa entre los electores sociales y los políticos.

Finalmente, este nuevo contexto ha obligado no solo a repensar las estrategias políticas en el siglo XXI, sino también a reconsiderar la forma de presentar al político y las relaciones que debe establecer con los ciudadanos para poder obtener su confianza y posterior voto. Por lo tanto, estas redes sociodigitales están modificando la forma de hacer política, por lo que su estudio poco a poco formará parte de área de conocimiento.

\section{Bibliografía}

Baldwin-Philippi, J. (2018). Politics 2.0: Social Media Campaining. En J. Burgess, A. Marwick, \& T. Poell (Eds.), The SAGE Handbook of Social Media (pp. 527-245). SAGE Publications.

Barabási, A.L. (2011). Introduction and Keynote to A Networked Self. En Z. Papacharissi, A Networked Self (pp. 1-14). Routledge.

Botometer (2020). Botometer. https://bit.ly/2MJOPy1

Cervantes, M. (@dodgeramlo) (25 de abril de 2020).Rt (tuit).https://bit.ly/3jCS2eA Boyd, D. (2011). Social Networks Sites as Networked Publics. En Z. Papacharissi, A Networked Self (pp. 39-58). Routledge.

Chávez, G. (2 de diciembre de 2020). Los bost de AMLo "inflan" su popularidad en las redes. Expansión. https://bit.ly/3tOyF6U 
Crovi-Druetta, D. (2012). Apropiación: una aproximación conceptual. En M. Portillo-Sánchez, \& I. Cornejo-Portugal (Coords.), ¿Comunicación posmasi$v a$ ? (pp. 149-162). Universidad Iberoamericana.

Dahlgren, P. (2012). Mejorar la participación: la democracia y el cambiante entorno de la web. En S. Champeau, \& D. Innerarity (Coords.), Internet y el futuro de la democracia (pp. 45-67). Paidós.

Derecho a la Red (6 de abril de 2020). Botometer, detecta si esa cuenta es un bot en Twitter. Derecho a la Red. https://bit.ly/3tL8b6r

Faesler, J.C. (@jcarlosfaesler) (12 de abril de 2020) Hoy es 12 de abril de 2020 (tuit). https://bit.ly/3qbhI4n

García-Calderón, C., \& Olmedo-Neri, R.A. (2019). El nuevo opio del pueblo: apuntes desde la Economía Política de la Comunicación para (des)entender la esfera digital. Iberoamérica Social, 7(XII), 84-96. https://bit.ly/3aQWPVO

Gómez-Castellanos, R.M., Ortiz-Marín, M., \& Concepción-Montiel, L.E. (2011). Tecnologías de la comunicación y política 2.0. Espacios Públicos, 14(30), 72-84. https://bit.ly/3tOrjjM

Grossi, G. (1985). La comunicación política moderna: entre partidos de masa y mass-media. En M. de Moragas, Sociología de la comunicación de masas (Vol. IV, pp. 144-164). Gustavo Gili.

Hanneman, R. (2000). Introducción a los métodos del análisis de redes sociales. Centro de Investigaciones Sociológicas.

Jorge, J.E. (2014). La comunicación política en las redes sociales. Enfoques teóricos y hallazgos empíricos. Questión. Revista Especializada en Periodismo y Comunicación, 1(44), 268-286. https://bit.ly/3aUc001

Lipovetsky, G. (1990). El imperio de lo efímero. Anagrama.

Lipovetsky, G., \& Serroy, J. (2009). La pantalla global. Anagrama.

Lozares, C., \& Verd, J.M. (2011). De la homofilia a la cohesión social y viceversa. Redes. Revista Hispana para el Análisis de Redes Sociales, 20, 29-50. https://doi.org/10.5565/rev/redes.408

Mathias, P. (2012). En las redes de Solón. Para una concepción cultural de la democracia digital. En S. Champeau, \& D. Innerarity, Internet y el futuro de la democracia (pp. 133-154). Paidós.

Olmedo-Neri, R.A. (2019). \#AmorEsAmor como constructor de redes digitales en el movimiento LGBTTTIQA en México. Virtualis, 10(19), 109-133. https://bit.ly/3jCTM7C

Olmedo-Neri, R.A. (2020). La comunicación en la posmodernidad: influencia en el sujeto, campo y medio. Iberoamérica Social (XIV), 157-172. https://bit. ly/3tLPNKI 
Paniagua-López, J.A. (2012). Curso de análisis de redes sociales. Metodología y estudios de caso. Universidad de Granada.

Peirone, F. (2012). Mundo extenso. FCE.

Quinn, K., \& Papacharissi, Z. (2018). Our Networked selves: Personal connection and relational maintenance in social media use. En J. Burguess, A. Marwick, \& T. Poell (Eds.), The SAGE Handbook of Social Media (pp. 353-371). SAGE Publications.

Mendieta-Ramírez, A., \& Estrada-Rodríguez, J.L. (2017). Comunicación política e informal en México: de la era televisiva al internet. Opción, 33(84), 154190. https://bit.ly/371wSBH

Reyes-Montes, M.C. (2007). Comunicación política y medios en México: el caso de la reforma a la Ley federal de Radio y televisión. Convergencia, 14(43), 105-136. https://bit.ly/2Ncwdqi

Reyes-Montes, M.C., O’Quínn-Parrales, J.A., \& Morales y Gómez, J.M. (2011). Reflexiones sobre la comunicación política. Espacios Públicos, 14(30), 85-101. https://bit.ly/3tMeYNm

Rogers, R. (2018). Digital methods for across-platform analysis. En J. Burgess, A. Marwick, \& T. Poell (Eds.), The SAGE Handbook of Social Media (pp. 91-110). SAGE Publications.

Sanz-Menéndez, L. (2003). Análisis de redes sociales o cómo representar las estructuras sociales subyacentes. Apuntes de Ciencia y Tecnología, 21-29. https://bit.ly/2N9WUvD

Tajonar, H. (18 de mayo de 2020). Bots en redes, ¿quién pompó? Aristegui Noticias https://bit.ly/3aUdZlj

Trejo-Delarbre, R. (2015). México enredado. Auge, ligereza y limitaciones en el uso político de las redes sociodigitales. En R. Winocur-Iparraguirre, \& J.A. Sánchez-Martínez (Coords.), Redes sociodigitales en México (pp. 17-39). FCE-CONACULTA.

Van Dijck, J. (2016). La cultura de la conectividad: Una historia crítica de las redes sociales. Siglo XXI Editores.

Fecha de envío: 2020/07/30; Fecha de aceptación: 2021/02/10;

Fecha de publicación: 2021/03/01 